\title{
Capillary Phase Transitions of $n$-Alkanes in a Carbon Nanotube
}

\author{
Jianwen Jiang, ${ }^{,, \dagger}$ Stanley I. Sandler, ${ }^{\dagger}$ and Berend Smit ${ }^{\ddagger}$ \\ Department of Chemical Engineering, University of Delaware, \\ Newark, Delaware 19716, and Department of Chemical Engineering, University of \\ Amsterdam, Nieuwe Achtergracht 166, 1018 WV Amsterdam, The Netherlands
}

Received October 30, 2003

\begin{abstract}
The capillary phase transitions of $n$-alkanes confined in a carbon nanotube are simulated for the first time using the gauge-cell Monte Carlo method with the configurational-bias scheme. At a subcritical temperature, the coexisting vapor-liquid phases are determined from a Maxwell construction along the adsorption isotherm, which exhibits a sigmoid van der Waals loop, including stable, metastable, and unstable regions. The confinement of an $n$-alkane in a carbon nanotube decreases its critical temperature, increases its critical density, and narrows its binodal curve.
\end{abstract}

There is considerable interest in the use of carbon nanotubes ${ }^{1}$ as adsorbents, membranes, and molecular sieves because of their unique internal nanometric structure. ${ }^{2}$ Molecular level computer simulation methods have been useful in investigating the properties of a fluid confined in nanotubes. Such methods provide a microscopic picture of a fluid in the interaction field of the confined space, and enable one to examine the underling physics. For example, there have been grand-canonical Monte Carlo (GCMC) and molecular dynamics simulations within carbon nanotubes of the adsorption of hydrogen,,$^{3-6}$ of the adsorption and diffusion of pure ${ }^{7}$ and mixed $^{8}$ Lennard-Jones fluids, and of the adsorption and separation of hydrogen isotopes. ${ }^{9}$ Much less explored are phase transitions inside carbon nanotubes. Fluids in nanopores exhibit a variety of states that differ from those of bulk fluids, such as layer transitions, freezing transitions, and capillary transitions, ${ }^{10}$ as a result of the spatial confinement and the competition between fluid-fluid and fluid-surface interactions. Using a simplified model of a carbon nanotube as a smooth structureless cylindrical pore, capillary phase transitions have been studied by $\mathrm{GCMC}^{11,12}$ and by Gibbs ensemble Monte Carlo (GEMC). ${ }^{13,14}$ However, the GCMC method cannot be used to directly simulate coexisting phases and needs to be supplemented with a thermodynamic integration technique to calculate the grand potential. The GEMC method involves volume changes of the simulation cells, which cannot be performed for a structured periodic adsorbent, such as the nanotube of this study.

* Corresponding author. E-mail: jiangj@che.udel.edu. Tel: (302)8316953. Fax: (302)831-1048.

University of Delaware.

$\doteqdot$ University of Amsterdam.
Recently the gauge-cell Monte Carlo simulation method has been proposed, ${ }^{15,16}$ which is superior to the GCMC and GEMC methods for the simulation of the complete adsorption isotherm, including the stable, metastable, and unstable regions. The coexisting phases can then be determined easily from the complete isotherm. However, thus far applications of the gauge-cell MC method have been focused on simple fluids, such as argon and nitrogen in smooth structureless nanopores. ${ }^{15,16}$ In this work, for the first time, we use the gauge-cell MC method to study the capillary phase transitions of a series of linear $n$-alkanes in a structured carbon nanotube, in which the carbon atom structure of the nanotube is included. This is different from previous studies, ${ }^{11-13,15,16}$ in which the nanotube was considered to be a smooth structureless nanocylinder. Also, the configurational-bias scheme, ${ }^{17-19}$ a recent advance in the simulation of chainlike molecules, has been employed. By this method a flexible molecule is grown atom-by-atom toward energetically favorable conformations, which is orders of magnitude more efficient than the traditional method of random growth. The configurational-bias scheme has been successfully applied in the studies of phase transitions and adsorption of alkanes and polymers. ${ }^{20-24}$

Model and Method. An open-ended $(30,30)$ single-walled carbon nanotube with a radius of $R=2.034 \mathrm{~nm}$ is chosen for this study; the extension to other carbon nanotubes is straightforward. Note that, however, in a very narrow nanotube the system approaches one-dimensional behavior and there is no phase transition. The carbon atoms along the nanotube are described explicitly, but they are frozen during simulation. The $n$-alkanes are represented by the united-atom model in which each $\mathrm{CH}_{3}$ or $\mathrm{CH}_{2}$ group is 
treated as a single interaction site. The bond length is rigid and fixed at $0.153 \mathrm{~nm}$. The interaction between the carbon atoms of the nanotube and each site of the alkane is modeled using the Lennard-Jones potential $4 \epsilon_{i j}\left[\left(\sigma_{i j} / r\right)^{12}-\left(\sigma_{i j} / r\right)^{6}\right]$, as is the nonbonded dispersive interaction between the sites on different alkane molecules or sites within an alkane molecule that are separated by four sites. The well depths $\epsilon_{i} / k_{\mathrm{B}}\left(k_{\mathrm{B}}\right.$ is the Boltzmann constant) and collision diameters $\sigma_{i}$ used are given in Table $1 .{ }^{25,26}$ The cross terms are obtained using the combining rules $\epsilon_{i j}=\sqrt{\epsilon_{i} \epsilon_{j}}$ and $\sigma_{i j}=\sqrt{\sigma_{i} \sigma_{j}}$. For propane and longer $n$-alkanes, the harmonic bond-bending potential ${ }^{27}$ along three successive sites is modeled using $0.5 k_{\theta}\left(\theta-\theta_{0}\right)^{2}$ with the force constant $k_{\theta} / k_{\mathrm{B}}=62500 \mathrm{~K} \mathrm{rad}^{-2}$ and the equilibrium bending angle $\theta_{0}=113.0^{\circ}$. For $n$-butane and longer $n$-alkanes, the dihedral torsion potential ${ }^{28}$ along four successive sites is modeled using $\sum_{k=0}^{3} v_{k} \cos (\phi)^{k}$ with the Fourier coefficients $v_{0} / k_{\mathrm{B}}=1009.728 \mathrm{~K}, v_{1} / k_{\mathrm{B}}=2018.446$ $\mathrm{K}, v_{2} / k_{\mathrm{B}}=136.341 \mathrm{~K}$, and $v_{3} / k_{\mathrm{B}}=-3164.520 \mathrm{~K}$.

The gauge-cell MC method can be viewed as a modification of GEMC. In GEMC the volumes of simulation cells change during simulation, which cannot be used here for the cell containing the periodic carbon nanotube. In the gauge-cell MC, two cells with fixed volumes are used. In this work, one cell contains the $(30,30)$ single-walled carbon nanotube of $3.074 \mathrm{~nm}$ in length, and periodic boundary condition is used in the axial dimension of the tube. The other cell is the so-called gauge cell, here a $5 \mathrm{~nm} \times 5 \mathrm{~nm} \times$ $5 \mathrm{~nm}$ cube with periodic boundary conditions in all three dimensions, containing the bulk fluid. The limited capacity in the gauge cell suppresses the density fluctuations of fluid in the nanotube and allows the fluid to be in a thermodynamically unstable state. The cutoff length used to evaluate the nonbonded Lennard-Jones energies was set to $1.537 \mathrm{~nm}$, half the nanotube length. A total of $2 \times 10^{7}$ cycles were used in the simulation, with the first $10^{7}$ cycles for equilibration, and the second $10^{7}$ cycles for obtaining ensemble averages. Each cycle consists of four types of trial moves for $n$-alkane molecules, including displacement, rotation, and regrowth of a randomly chosen molecule in each cell, and the swap of a molecule between the two cells. Equivalent to the Widom test particle method, ${ }^{29}$ from the swap the chemical potential of the bulk fluid in the gauge cell is estimated at no extra computational cost. This procedure is faster than previous gauge-cell simulations, ${ }^{15,16}$ in which the chemical potential in the gauge cell was determined from a series of separate GCMC simulations. In addition, the configurationalbias scheme ${ }^{17-19}$ has been used to speed up the sampling of $n$-alkane molecules in the confined environment. A molecule is grown atom-by-atom trying not to overlap with the other atoms. In doing so, $m(i=1 \ldots m, m$ was set to six in this work) random trial positions are generated with a probability proportional to $\exp \left(-\beta U_{i}^{\text {int }}\right)$, where $\beta=1 / k_{\mathrm{B}} T$ and $U_{i}^{\text {int }}$ is the internal energy at a position $i$ including the intramolecular bond-bending and torsion potentials. From among these trial positions, one is selected with a probability $\exp \left(-\beta U_{i}^{\text {ext }}\right) /$ $\sum_{i} \exp \left(-\beta U_{i}^{\mathrm{ext}}\right)$, where $U_{i}^{\mathrm{ext}}$ is the external energy including all nonbonded Lennard-Jones potentials. During this growing

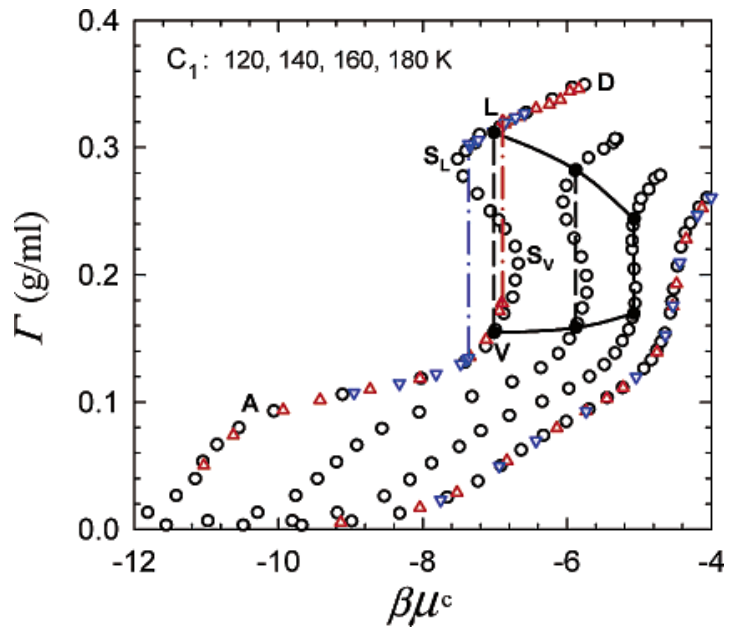

Figure 1. Isotherms of methane $\left(C_{1}\right)$ in the nanotube at 120, 140, 160 , and $180 \mathrm{~K}$. The open circles are from the gauge-cell MC. The solid circles connected by the dashed lines are coexisting vapor and liquid phases. The solid curve is binodal curve. The regions of $\mathrm{AV}$ and DL are stable, of $\mathrm{VS}_{\mathrm{V}}$ and $\mathrm{LS}_{\mathrm{L}}$ are metastable, and of $\mathrm{S}_{\mathrm{V}} \mathrm{S}_{\mathrm{L}}$ is unstable. At 120 and $180 \mathrm{~K}$, the red upper and blue lower triangles are the adsorption and desorption branches, respectively, from GCMC. At $120 \mathrm{~K}$, the red and blue dot-dashed lines indicate capillary condensation and evaporation, respectively, from GCMC.

Table 1: Nonbonded Lennard-Jones Potential Parameters

\begin{tabular}{lrc}
\hline site-site & $\epsilon / \mathrm{K}_{\mathrm{B}}(\mathrm{K})$ & $\sigma(\mathrm{nm})$ \\
\hline $\mathrm{C}-\mathrm{C}^{25}$ & 28.0 & 0.340 \\
$\mathrm{CH}_{4}-\mathrm{CH}_{4}{ }^{26}$ & 148.0 & 0.373 \\
$\mathrm{CH}_{3}-\mathrm{CH}_{3}{ }^{26}$ & 98.0 & 0.375 \\
$\mathrm{CH}_{2}-\mathrm{CH}_{2}{ }^{26}$ & 46.0 & 0.395
\end{tabular}

process a bias is introduced and is removed by adjusting the acceptance rules.

Results. Figure 1 shows the isotherms of methane in the $(30,30)$ carbon nanotube at $120,140,160$, and $180 \mathrm{~K}$ as a function of bulk configurational chemical potential $\beta \mu^{\mathrm{c}}$. The extent of adsorption is given as the overall adsorbate density inside the nanotube defined as $\Gamma=2 \int_{0}^{R} \rho(r) r \mathrm{~d} r / R^{2}$, where $\rho(r)$ is the local density at a position $r$. The open circles in the figure are the results from the gauge-cell MC simulation. At a subcritical temperature, we obtain complete isotherm with a van der Waals loop, which is related to capillary phase transition. The coexisting vapor-liquid phases are determined along the isotherm using a Maxwell equal area construction $\int_{\mu_{\mathrm{V}}}^{\mu_{\mathrm{S} V}} \Gamma_{\mathrm{A}}(\mu) \mathrm{d} \mu-\int_{\mu_{\mathrm{SL}}}^{\mu_{\mathrm{S}}} \Gamma_{\mathrm{S}}(\mu) \mathrm{d} \mu+\int_{\mu_{\mathrm{SL}}}^{\mu_{\mathrm{L}}} \Gamma_{\mathrm{D}}(\mu) \mathrm{d} \mu$ $=0$, where $\mathrm{V}$ and $\mathrm{L}$ are vapor and liquid equilibrated phases, $\mathrm{S}_{\mathrm{V}}$ and $\mathrm{S}_{\mathrm{L}}$ are vapor and liquid spinodals. The regions of $\mathrm{AV}$ and DL are stable, of $\mathrm{VS}_{\mathrm{V}}$ and $\mathrm{LS}_{\mathrm{L}}$ are metastable, and of $\mathrm{S}_{\mathrm{V}} \mathrm{S}_{\mathrm{L}}$ is unstable. The coexisting phases determined are shown in the figure as the solid circles linked by the dashed tie line. The solid curve is the binodal curve for methane confined in the nanotube drawn through the coexisting phases at 120,140 , and $160 \mathrm{~K}$. However, at $180 \mathrm{~K}$, the isotherm is a monotonic function of chemical potential without a van der Waals loop, which implies that $180 \mathrm{~K}$ is higher than the critical temperature of methane in the nanotube. Note that the critical temperature of bulk methane is $190.6 \mathrm{~K}$. 


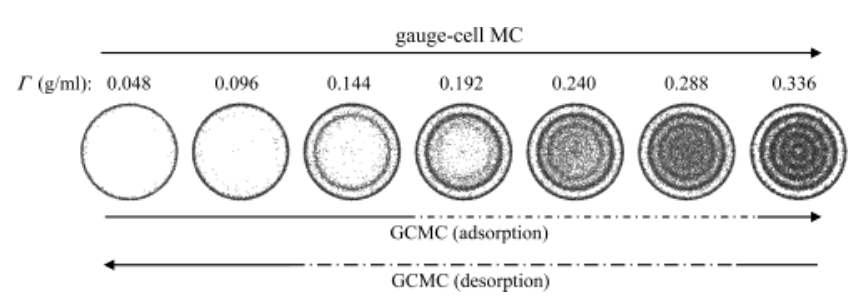

Figure 2. Snapshots of methane in the nanotube at $120 \mathrm{~K}$ at various adsorbed densities $0.048,0.096,0.144,0.192,0.240,0.288$, and $0.336(\mathrm{~g} / \mathrm{mL})$. The arrow at the top illustrates the process from the gauge-cell MC. The arrows at the bottom illustrate the processes of adsorption and desorption, respectively, from GCMC with the dot - dashed lines indicate inaccessible regions.

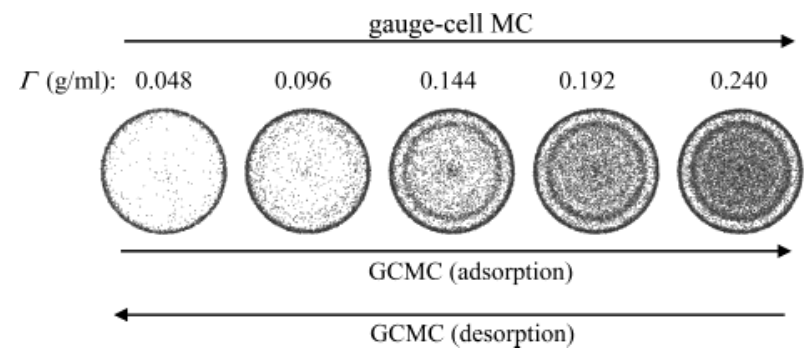

Figure 3. Snapshots of methane in the nanotube at $180 \mathrm{~K}$ at various adsorbed densities 0.048, 0.096, 0.144, 0.192, and $0.240(\mathrm{~g} / \mathrm{mL})$. The arrow at the top illustrates the process from the gauge-cell MC. The arrows at the bottom illustrate the processes of adsorption and desorption, respectively, from GCMC.

For comparison, GCMC simulations have also been carried out at two temperatures, 120 and $180 \mathrm{~K}$. The red upper and blue lower triangles are for adsorption and desorption, respectively. At $120 \mathrm{~K}$, capillary condensation and evaporation (red and blue dot-dashed lines) are clearly observed from the GCMC simulation with an IUPAC classified H1 type hysteresis. Hysteresis in the adsorption-desorption loop is associated with the mechanism of pore filling-emptying by capillary condensation-evaporation. The coexisting phases determined using the gauge-cell $\mathrm{MC}$ are between the capillary condensation and evaporation, i.e., inside the hysteresis loop. Though the phase transition should be located within the hysteresis loop, to determine the exact position from GCMC is not an easy task. With a very long GCMC run, the hysteresis loop might vanish so that the phase transition points could be determined. The advantage of the gauge-cell MC method is that the phase transition points can be determined easily, in addition to obtaining the complete adsorption isotherm.
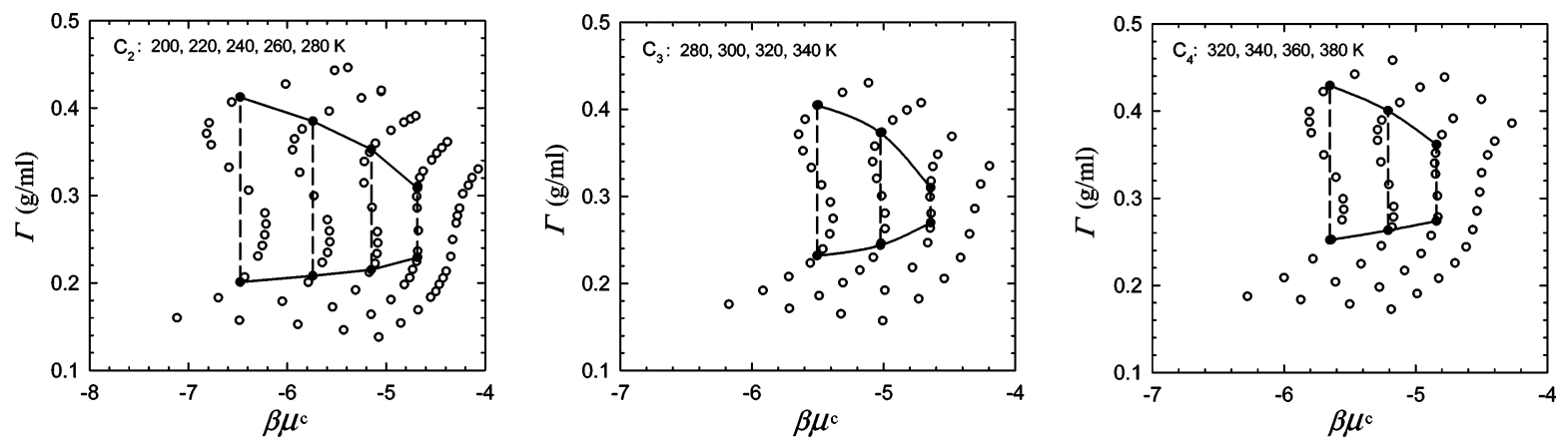

Figure 4. Isotherms of ethane $\left(\mathrm{C}_{2}\right)$, propane $\left(\mathrm{C}_{3}\right)$, and $n$-butane $\left(\mathrm{C}_{4}\right)$ in the nanotube at various temperatures, the legend as in Figure 1 .
In the stable regions $\mathrm{AV}$ and $\mathrm{DL}$, and part of the metastable regions $\mathrm{VS}_{\mathrm{V}}$ and $\mathrm{LS}_{\mathrm{L}}$ where GCMC can be used, it gives results identical to those obtained with the gaugecell MC. However, GCMC cannot access the unstable region $S_{V} S_{L}$. It is expected that the hysteresis loop obtained from GCMC simulation would be reduced in size on increasing temperature from 120 to $140 \mathrm{~K}$ and then $160 \mathrm{~K}$. At $180 \mathrm{~K}$, the GCMC results do not exhibit hysteresis and are in agreement with the gauge-cell $\mathrm{MC}$ results, further verifying that there is no phase transition at $180 \mathrm{~K}$ and that the critical temperature of methane confined in this carbon nanotube is below $180 \mathrm{~K}$. Above the capillary critical temperature, the isotherm is reversible, indicating that the distinction between the vapor and condensed liquid phases disappears.

Figure 2 shows the snapshots of methane in the nanotube at $120 \mathrm{~K}$ at various adsorbed densities, from $0.048,0.096$, $0.144,0.192,0.240,0.288$, to $0.336(\mathrm{~g} / \mathrm{mL})$ obtained by accumulating 50 widely spaced equilibrium configurations (nanotube not shown). At the lowest density, the preferred location of the methane molecules is in a single annular layer near the nanotube wall. With increasing density, the number of layers increases up to five, with each subsequent layer closer to the nanotube center. The solid-line arrow at the top of the figure is used to indicate that the gauge-cell MC method can be used to simulate the complete isotherm from stable to metastable and then to the unstable region. The broken-line arrows at the bottom of the figure represent the adsorption and desorption processes, respectively, that can be accessed by using GCMC. The dot-dashed portions of the lines indicate those regions inaccessible by GCMC. Consequently, from GCMC, one is unable to observe the snapshots with three and four annular layers along either the adsorption or desorption path. Capillary condensation or evaporation is commonly regarded as a secondary process, which is always preceded by adsorption or desorption, and a hysteresis loop usually appears in the multilayer range of a physisorption isotherm. All of these are seen in the sequence of simulation snapshots.

Figure 3 shows the snapshots of methane locations in the nanotube at various adsorbed densities, from 0.048, 0.096, $0.144,0.192$, to $0.240(\mathrm{~g} / \mathrm{mL})$, but at $180 \mathrm{~K}$ (nanotube not shown). At the lowest density, there is one annular layer near the nanotube wall, and with increasing density, the number of layers increases. Compared with the snapshots at $120 \mathrm{~K}$, the distribution of methane molecules inside the 


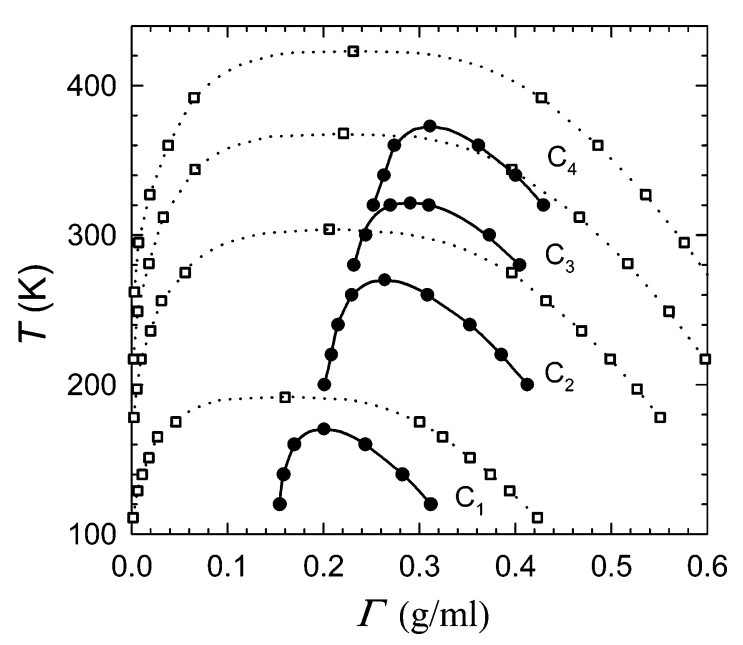

Figure 5. Binodal curves for the confined and bulk methane, ethane, propane, and $n$-butane. The circles are for the confined alkanes, and the squares ${ }^{26}$ are for the bulk alkanes.

nanotube is more dispersed, as is expected due to increased thermal motion. Since $180 \mathrm{~K}$ is above the critical temperature of capillary phase transition, the gauge-cell MC and GCMC give same results, and the complete isotherm and series of snapshots can be obtained by both simulation methods.

Figure 4 shows the predicted isotherms for ethane, propane, and $n$-butane in the nanotube at various temperatures obtained using the gauge-cell MC method. For each alkane, adsorption is simulated at several subcritical temperatures, and the vapor-liquid coexisting points determined; in each case the highest temperature is above the critical temperature of the confined alkane, and the isotherm does not possess a van der Waals loop.

Figure 5 compares the binodal curves for confined and bulk methane, ethane, propane, and $n$-butane. The circles and solid lines are for the confined alkanes from this work, and the squares and dotted lines are for the bulk alkanes obtained using GEMC simulations. ${ }^{26}$ The critical temperatures $T_{\mathrm{c}}$ and densities $\Gamma_{\mathrm{c}}$ have been estimated by fitting the coexisting data to $\Gamma_{ \pm}=\Gamma_{\mathrm{c}}+A\left(T_{\mathrm{c}}-T\right) \pm 0.5 B\left(T_{\mathrm{c}}-T\right)^{\beta}$ based on renormalization-group theory, ${ }^{30}$ where + denotes liquid phase and - denotes vapor phase. The amplitudes $A$ and $B$ and the critical exponent $\beta$ are treated as adjustable parameters. Compared with the bulk alkane, for the confined alkane the capillary critical temperature is consistently lower, the critical density is higher, and the binodal curve is narrower.

Summary. The capillary phase transitions of $n$-alkanes in a carbon nanotube have been investigated for the first time using the novel gauge-cell MC simulation with the configurational-bias scheme. By obtaining the complete isotherm, including the stable, metastable, and unstable regions, the coexisting phases were determined. In the regions in which GCMC was applicable, consistent results between the gaugecell MC and GCMC were obtained. The capillary phase transition can be considered to be a surface-driven phase transition shifted from that of the bulk fluid resulting in a decreased critical temperature, an increased critical density, and a narrower binodal curve. This work presents the results for the short $n$-alkanes from methane to $n$-butane; the extension to longer $n$-alkanes is underway.

Acknowledgment. J.J. and S.I.S. acknowledge the support of the U.S. National Science Foundation under Grant EEC-0085461 and the U.S. Department of Energy under Grant DE-FG02-85ER13436. B.S. acknowledges the support of The Netherlands Research Council for Chemical Sciences (CW) through PIONIER.

\section{References}

(1) Iijima, S. Nature 1991, 354, 56.

(2) Harris, P. J. F. Carbon Nanotubes and Related Structures: New Materials for the 21st Century; Cambridge University Press: Cambridge, UK, 1999.

(3) Rzepka, M.; Lamp, P.; de la Casa-Lillo, M. A. J. Phys. Chem. B 1998, 102, 10894.

(4) Cracknell, R. F. Mol. Phys. 2002, 100, 2079.

(5) Ma, Y. C.; Xia, Y. Y.; Zhao, M. W.; Ying, M. J. Phys. Rev. B 2002 65 , art. no.

(6) Gu, C.; Gao, G. H. Phys. Chem. Chem. Phys. 2002, 4, 4700.

(7) Duren, T.; Keil, F. J.; Seaton, N. A. Mol. Phys. 2002, 100, 3741.

(8) Ayappa, K. G. Langmuir 1998, 14, 880.

(9) Challa, S. R.; Sholl, D. S.; Johnson, J. K. J. Chem. Phys. 2002, 116, 814.

(10) Gelb, L. D.; Gubbins, K. E.; Radhakrishnan, R.; Sliwinska-Bartkowiak, M. Rep. Prog. Phys. 1999, 62, 1573.

(11) Peterson, B. K.; Gubbins, K. E. Mol. Phys. 1987, 62, 215.

(12) Maddox, M. W.; Gubbins, K. E. Langmuir 1995, 11, 3988.

(13) Panagiotopoulos, A. Z. Mol. Phys. 1987, 62, 701.

(14) Brovchenko, I.; Geiger, A.; Oleinikova, A. Phys. Chem. Chem. Phys. 2001, 3, 1567.

(15) Neimark, A. V.; Vishnyakov, A. Phys. Rev. E 2000, 62, 4611.

(16) Vishnyakov, A.; Neimark, A. V. J. Phys. Chem. B 2001, 105, 7009.

(17) Siepmann, J. I.; Frenkel, D. Mol. Phys. 1992, 75, 59.

(18) Frenkel, D.; Mooij, G. C. A. M.; Smit, B. J. Phys.: Condens. Matter 1992, 4, 3053.

(19) de Pablo, J. J.; Laso, M.; Suter, U. W. J. Chem. Phys. 1992, 96, 2395.

(20) Siepmann, J. I.; Karaborni, S.; Smit, B. Nature 1993, 365, 330.

(21) Smit, B.; Siepmann, J. I. Science 1994, 264, 1118.

(22) Smit, B.; Maesen, T. L. M. Nature 1995, 374, 42.

(23) Jiang, J. W.; Yan, Q. L.; Liu, H. L.; Hu, Y. Macromolecules 1997. $30,8459$.

(24) Jiang, J. W.; Liu, H. L.; Hu, Y. Macromol. Theor. Simul. 1998, 7 , 105.

(25) Steele, W. A. The Interaction of Gases with Solid Surfaces; Pergamon: Oxford, 1974.

(26) Martin, M. G.; Siepmann, J. I. J. Phys. Chem. B 1998, 102, 2569.

(27) van der Ploeg, P.; Berendsen, H. J. C. J. Chem. Phys. 1982, 76, 3271.

(28) Wang, Y.; Hill, K.; Harris, J. G. J. Chem. Phys. 1993, 100, 3276.

(29) Widom, B. J. Chem. Phys. 1963, 39, 2802.

(30) Domb, C. The Critical Point: A Historical Introduction to the Modern Theory of Critical Phenomena; Taylor \& Francis: London, 1996.

NL034961Y 